\title{
Magnetoresistance in Graphene-Based Ferromagnetic/ Rashba Barrier/Ferromagnetic Heterojunction
}

\author{
H. Mohammadpour and K. Hasanirokh \\ Azarbaijan Shahid Madani University, 53714-161 Tabriz, Iran \\ (Received May 3, 2015; in final form December 28, 2015)
}

\begin{abstract}
The quantum tunnelling of spin-polarized electrons through a Rashba barrier on a single layer graphene is studied by the scattering matrix method. It is shown that the magnetoresistance, defined as the difference between conductance at the presence and absence of the Rashba spin-orbit interaction, oscillates with the intensity of interaction. These oscillations are also observed in the conductance versus the potential energy of the barrier.
\end{abstract}

DOI: 10.12693/APhysPolA.129.75

PACS: 72.80.Vp, 73.23.-b, 73.22.Pr

\section{Introduction}

The long-range spin-coherence length beside unique electronic properties, make graphene a promising candidate for application in spintronic along with the nanoelectronic devices [1-3]. Application of graphene in spintronic is mainly manipulated by the control of spin-orbit interactions (SOI) [4-6]. The SOI in graphene comprises of intrinsic and extrinsic (Rashba) components $[7,8]$. The former is induced by the carbon intra-atomic interactions and is very weak in a free-standing graphene [9]. Therefore, we have neglected it in this work. The Rashba SOI that arises due to the structural inversion asymmetry (SIA) in the system, introduces an effective magnetic field that can be controlled e.g. by applying a gate voltage on top of the graphene sheet.

The magnetotransport at quantum-sized structures has been widely studied theoretically and experimentally. In graphene-based systems, the quantum oscillations of the spin-dependent conductance and the magnetoresistance have been studied in normal-ferromagnetic junctions [10-12].

It should be noted that the term "magnetoresistance" stands usually for the effect of external magnetic fields or intrinsic magnetization on the resistivity. However it is also applied to the contribution to the conductance of other spin-dependent interactions such as magnetic impurities and spin-orbit coupling [13]. In this paper, by employing the second definition, we have used the "magnetoresistance" as the effect of the SOI on the conductance of system.

We have modeled a graphene-based ferromagnetic (FG)-normal (NG) three-layer heterostructure as $\mathrm{FG} / \mathrm{NG} / \mathrm{FG}$, in which the normal region is sandwiched between two ferromagnetic semi-infinite layers (contacts). The conductance of system is calculated in the presence of a Rashba barrier of various intensities.

\section{Model and approach}

The planar FG/NG/FG structure is assumed to be in the $x-y$ plane [14]. The $y$-direction is parallel to the interfaces and the $x$-direction is normal to it (Fig. 1). We have

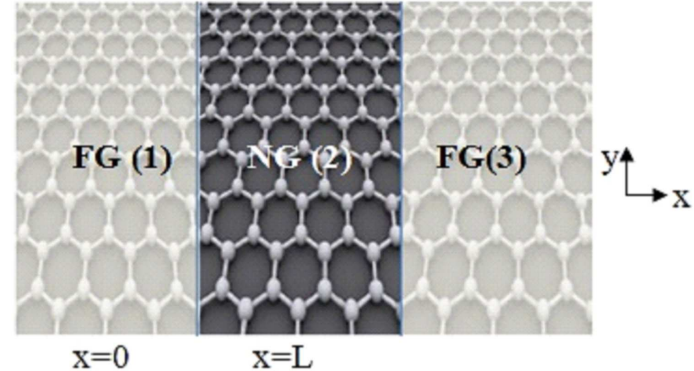

Fig. 1. Schematic image of the model system.

assumed large width of the sheet along the $y$-direction such that we can neglect the edge effects.

Scattering formalism is employed for calculating the transport amplitudes of electrons.

The Hamiltonian in regions 1 and 3 of Fig. 1 is given by

$$
\hat{H}=\hat{H}_{0}+\hat{H}_{S} \text {. }
$$

$\hat{H}_{0}$ is the Dirac Hamiltonian for massless fermions [15]:

$$
\hat{H}_{0}=-\mathrm{i} \hbar v_{\mathrm{F}}\left(\hat{\sigma}_{x} \partial_{x}+\hat{\sigma}_{y} \partial_{y}\right) \otimes \hat{I}_{S} .
$$

Here $\hbar$ is the Planck constant and $v_{\mathrm{F}}$ represents the Fermi velocity in graphene. $\hat{\sigma}$ stands for the Pauli matrix indicating the states on the A and B sublattices of graphene and $\hat{I}_{S}$ is the unit operator in the spin space.

$\hat{H}_{S}$ denotes the exchange Hamiltonian in ferromagnetic regions (1) and (3):

$$
\hat{H}_{S}(1)=h \hat{\tau}_{z}, \quad \hat{H}_{S}(3)=h^{\prime} \hat{\tau}_{z} .
$$

$\tau_{z}$ represents the Pauli matrix in spin space and $h, h^{\prime}$ are the exchange parameters of layers 1 and 3 , respectively.

The Hamiltonian in the Rashba region (NG) is

$$
\hat{H}=\hat{H}_{0}+\hat{H}_{R} .
$$

$\hat{H}_{R}$ is the Rashba SOI, and can be written as $[16,17]$ :

$$
\hat{H}_{R}=\left(\frac{\lambda_{R}}{2}\right)\left(\hat{\sigma}_{y} \hat{\tau}_{x}-\hat{\sigma}_{x} \hat{\tau}_{y}\right),
$$

here $\lambda_{R}$ is the Rashba SOI constant. (Note: Because of the valley degeneracy in the modelled system of graphene, Hamiltonians above are written in just one valley ignoring the valley-dependence of the operators.) 
We consider a spin-up electron with energy $E$ incident from the left FG to the interface with the Rashba region. The solutions of Dirac Eqs. (1) and (4), are as the following 4-component pseudospinors, with amplitude normalized to 1 , in which the upper two entities refer to the two sub-lattice components of spin up type and the other two indicate the spin down components

$$
\begin{gathered}
\psi(x \leq 0)=\exp (\mathrm{i} k x \cos \phi)\left(\begin{array}{l}
\mathrm{e}^{-\mathrm{i} \phi / 2} \\
\mathrm{e}^{\mathrm{i} \phi / 2} \\
0 \\
0
\end{array}\right) \\
+r \exp (-\mathrm{i} k x \cos \phi)\left(\begin{array}{l}
\mathrm{e}^{-\mathrm{i}(\pi-\phi) / 2} \\
\mathrm{e}^{\mathrm{i}(\pi-\phi) / 2} \\
0 \\
0
\end{array}\right) \\
+r^{\prime} \exp \left(-\mathrm{i} k^{\prime} x \cos \phi^{\prime}\right)\left(\begin{array}{l}
0 \\
0 \\
\mathrm{e}^{-\mathrm{i}\left(\pi-\phi^{\prime}\right) / 2} \\
\mathrm{e}^{\mathrm{i}\left(\pi-\phi^{\prime}\right) / 2}
\end{array}\right), \\
k=\frac{E+h}{\hbar v_{f}}, \quad k^{\prime}=\frac{E-h}{\hbar v_{f}}, \quad \phi^{\prime}=\sin ^{-1}\left(k \tan \phi / k^{\prime}\right) .
\end{gathered}
$$

The electron beam at the 1st FG region makes an angle $\phi$ (for spin up) and angle $\phi^{\prime}$ (for spin down) with the $x$ axis. The angles of the spin-mixed wave functions at the NG region with Rashba SOI are $\varphi_{1}, \varphi_{2}, \varphi_{3}$, and $\varphi_{4}$ and in the 2nd FG region they are $\bar{\phi}$ (for spin up) and $\bar{\phi}^{\prime}$ (for spin down).

We assume a Fermi energy mismatch between the normal region and the ferromagnetic layers and denote it with $U$.

The wave function in the Rashba region then reads

$$
\begin{aligned}
& \psi(0 \leq x \leq L)= \\
& A \exp \left(\mathrm{i} k_{1} x \cos \phi_{1}\right)\left(\begin{array}{l}
\frac{\hbar v_{\mathrm{F}} k}{E} \mathrm{e}^{-\mathrm{i} \phi_{1}} \\
1 \\
\frac{-\frac{\hbar^{2} v_{\mathrm{F}}^{2} k_{1}^{2}}{E}+E}{\mathrm{i} \lambda_{R}} \\
\frac{\hbar v_{\mathrm{F}} k_{1}}{\mathrm{i} \lambda_{R} E}\left(E-\frac{\hbar^{2} v_{\mathrm{F}}^{2} k_{1}^{2}}{E}\right) \mathrm{e}^{\mathrm{i} \phi_{1}}
\end{array}\right) \\
& +B \exp \left(\mathrm{i} k_{2} x \cos \phi_{2}\right)\left(\begin{array}{l}
\mathrm{e}^{-\mathrm{i} \phi_{2} \frac{\hbar v_{\mathrm{F}} k_{2}}{E}} \\
1 \\
\frac{E-\frac{\hbar^{2} v_{\mathrm{F}}^{2} k_{2}^{2}}{E}}{\mathrm{i} \lambda_{R}} \\
\frac{\hbar v_{\mathrm{F}} k_{2}}{\mathrm{i} \lambda_{R} E}\left(E-\frac{\hbar^{2} v_{\mathrm{F}}^{2} k_{2}^{2}}{E}\right) \mathrm{e}^{\mathrm{i} \phi_{2}}
\end{array}\right) \\
& +C \exp \left(\mathrm{i} k_{3} x \cos \phi_{3}\right)\left(\begin{array}{l}
\frac{\hbar v_{\mathrm{F}} k_{3}}{E} \mathrm{e}^{-\mathrm{i} \phi_{3}} \\
1 \\
\frac{E-\frac{\hbar^{2} v_{\mathrm{F}}^{2} k_{3}^{2}}{E}}{\mathrm{i} \lambda_{R}} \\
\frac{\hbar v_{\mathrm{F}} k_{3}}{\mathrm{i} \lambda_{R} E}\left(E-\frac{\hbar^{2} v_{\mathrm{F}}^{2} k_{3}^{2}}{E}\right) \mathrm{e}^{\mathrm{i} \phi_{4}}
\end{array}\right)
\end{aligned}
$$

$$
+D \exp \left(\mathrm{i} k_{4} x \cos \phi_{4}\right)\left(\begin{array}{l}
\frac{\hbar v_{\mathrm{F}} k_{4}}{E} \mathrm{e}^{-\mathrm{i} \phi_{4}} \\
1 \\
\frac{E-\frac{\hbar^{2} v_{\mathrm{F}}^{2} k_{4}^{2}}{E}}{\mathrm{i} \lambda_{R}} \\
\frac{\hbar v_{\mathrm{F}} k_{4}}{\mathrm{i} \lambda_{R} E}\left(E-\frac{\hbar^{2} v_{\mathrm{F}}^{2} k_{4}^{2}}{E}\right) \mathrm{e}^{\mathrm{i} \phi_{4}}
\end{array}\right),
$$

where

$$
\begin{array}{ll}
k_{1}=\frac{\sqrt{(E+U)^{2}-\lambda_{R}(E+U)}}{\hbar v_{\mathrm{F}}}, & k_{3}=-k_{1}, \\
k_{2}=\frac{\sqrt{(E+U)^{2}+\lambda_{R}(E+U)}}{\hbar v_{\mathrm{F}}}, & k_{4}=-k_{2}, \\
\phi_{1}=\tan ^{-1}\left(k \tan \phi / k_{1}\right), & \\
\phi_{2}=\tan ^{-1}\left(k \tan \phi / k_{2}\right), & \\
\phi_{3}=\pi-\phi_{1}, \quad \phi_{4}=\pi-\phi_{2} . &
\end{array}
$$

$r, t, r^{\prime}, t^{\prime}$ are the scattering amplitudes in regions 1 and 3 while $A, B, C$, and $D$ are the coefficients of the electron wave function in the middle layer.

In the region 3 the wave function takes the form

$$
\begin{gathered}
\psi(x \geq L)=t \exp (\mathrm{i} \bar{k} x \cos \bar{\phi})\left(\begin{array}{l}
\mathrm{e}^{-\mathrm{i} \bar{\phi} / 2} \\
\mathrm{e}^{\mathrm{i} \bar{\phi} / 2} \\
0 \\
0
\end{array}\right) \\
+t^{\prime} \exp \left(-\mathrm{i} \bar{k}^{\prime} x \cos \bar{\phi}^{\prime}\right)\left(\begin{array}{l}
0 \\
0 \\
\mathrm{e}^{-\mathrm{i} \bar{\phi}^{\prime} / 2} \\
\mathrm{e}^{\mathrm{i} \bar{\phi}^{\prime} / 2}
\end{array}\right) . \\
\bar{k}=\frac{E+h^{\prime}}{\hbar v_{\mathrm{F}}}, \quad \bar{k}^{\prime}=\frac{E-h^{\prime}}{\hbar v_{\mathrm{F}}} .
\end{gathered}
$$

From conservation of the $y$-component of wave vector one obtains

$$
k \sin \phi=k_{i} \sin \phi_{i} .
$$

The transmission amplitudes for spin up $(t)$ and spin down $\left(t^{\prime}\right)$ electrons are obtained by applying continuity of the spinor components of the wave function at interfaces as below [18]:

$$
\psi\left(0^{-}\right)=\psi\left(0^{+}\right), \quad \psi\left(L^{-}\right)=\psi\left(L^{+}\right) .
$$

The conductance for spin up incident electrons is given by [19]:

$$
G=\int\left[T(\phi)_{(\uparrow \rightarrow \uparrow)}+T(\phi)_{(\uparrow \rightarrow \downarrow)}\right] \cos (\phi) \mathrm{d} \phi,
$$

where $T(\phi)_{(\uparrow \rightarrow \uparrow)}=t^{*} t$ and $T(\phi)_{(\uparrow \rightarrow \downarrow)}=t^{*} t^{\prime}$ are spindependent transmissions and the magnetoresistance is defined as:

$$
M R(\lambda)=\frac{G(\lambda=0)-G(\lambda)}{G(\lambda)} .
$$

\section{Results and discussion}

Numerical study of the spin transport properties in the system $\mathrm{FG} / \mathrm{NG} / \mathrm{FG}$ is presented in this section. In all the 
calculations, the Fermi energy is ed to be located at $E_{\mathrm{F}}=$ $1 \mathrm{meV}$ [17] and all energies are written in units of $E_{\mathrm{F}}$ and the lengths are in units of the Fermi wavelength, $\lambda_{\mathrm{F}}$.

It should be mentioned at the outset that it is assumed that the incoming electron beam is polarized in up spin state. We first consider the effect of the Fermi energy mismatch, $U$, on the conductance.

In Fig. 2, the conductance, $G$, is plot as a function of $U$ for $h=-0.1$ and $h^{\prime}=-0.4$ (in units of $E_{\mathrm{F}}$ ) and different Rashba intensities. The length of the Rashba region, $L$, is taken to be 50 (in units of $\lambda_{\mathrm{F}}$ ).

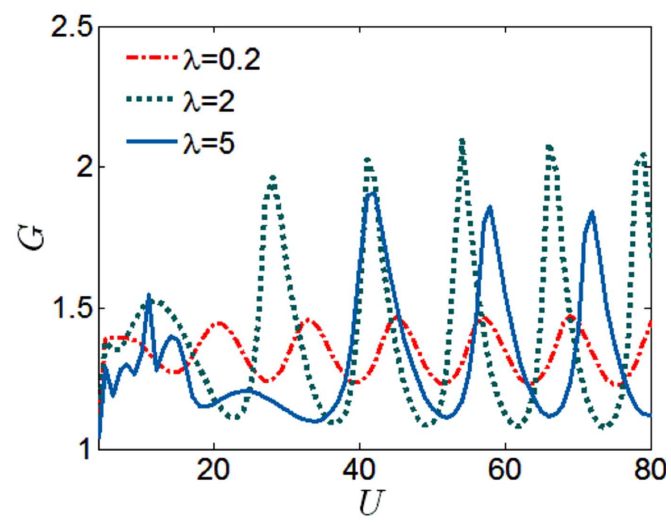

Fig. 2. $G$ as a function of $U$ for $h=-0.1$ and $h^{\prime}=-0.4$ and different $\lambda$ for $L=50$.

The well known quantum oscillations of the conductance with $U$ are revealed. The difference between curves are clearly footprints of the Rashba interaction. For small amounts of $U$, the Rashba coupling gives rise to a disordered conductance.

The same phenomena of quantum oscillations of $G$ versus $U$ are observed in Fig. 3 for different values of $L$.

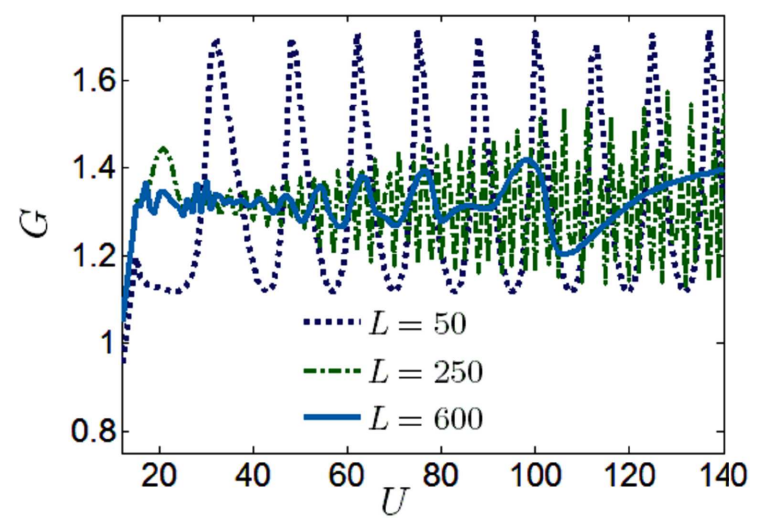

Fig. 3. $G$ as a function of $U$ for $h=-0.1, h^{\prime}=-0.3$, $\lambda=4$.

By increasing $L$, the amplitude of the oscillations decreases while the average magnitude of $G$ is preserved. The length-independent (average of) $G$ is a characteristic of the ballistic transport.
The resultant magnetoresistances (MR) which are derived from Eq. (13) are plotted in Figs. 4 to 6. In Fig. 4, the MR is plotted versus $\lambda$ for two amounts of $L=50$ and $L=100$.

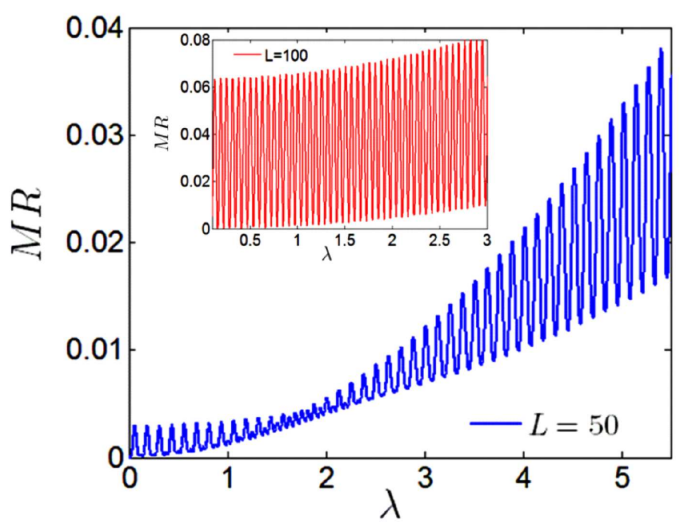

Fig. 4. MR as a function of Rashba coupling for $U=$ $2000, h=-0.1, h^{\prime}=-0.3$.

According to Fig. 4, MR of the system reveals strong oscillations with the Rashba coupling while it is controlled by the length of the Rashba region $(L)$. By comparing the main plot with the inset diagram it is deduced that by decreasing $L$, the magnitude of MR decreases and becomes strongly $\lambda$ - dependent.

At the same time, as shown in Fig. 5, the oscillatory behaviour of MR by $\lambda$ can be effectively changed by the value of $U$.

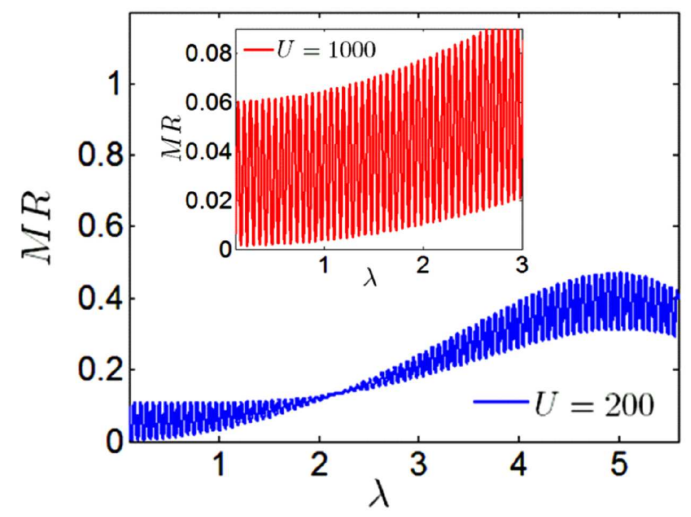

Fig. 5. MR as a function of Rashba coupling for $h=$ $-0.1, h^{\prime}=-0.3, L=100$.

As illustrated in Fig. 5, at smaller $U$ both of the magnitude and the $\lambda$-dependence of MR increase.

The MR is depicted in Fig. 6 as a function of $\lambda$ for two amounts of $h^{\prime}(=-0.2,-0.8)$ and other parameters written in the caption.

The increased MR at $h^{\prime}=-0.2$ compared to $h^{\prime}=-0.8$ can be explained based on the fact that by increasing $h^{\prime}$, the FG (3) supports mainly only one kind of spin subband and in this way prohibits the other spin sub-band 


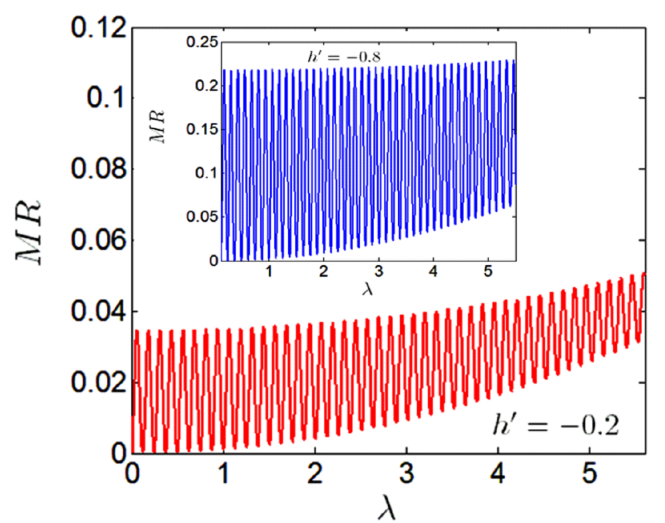

Fig. 6. The MR as a function of Rashba coupling for $U=2000, h=-0.1, L=50$.

from the transport and results in decreased MR. It is worth to mention that this increase has a maximum at about $h^{\prime}=1$ above which the respective spin sub-bands density of states are reversed.

\section{Conclusion}

In this paper the magnetotransport in graphene-based FG/NG/FG has been modelled for studying the effect of Rashba spin-orbit interaction on the conductance and magnetoresistance.

Results show that beside the critical role played by the Rashba SOI, other parameters, i.e., $U, h^{\prime}$ and $L$ can also effectively control the transport properties.

\section{Acknowledgments}

This research was supported by a grant fund number 217/D/8715 from Azarbaijan Shahid Madani University.

\section{References}

[1] K.S. Novoselov, A.K. Geim, S.V. Morozov, D. Jiang, Y. Zhang, S.V. Dubonos, I.V. Grigorieva, A.A. Firsov, Science 306, 666 (2004).

[2] P.R. Wallace, Phys. Rev. 71, 622 (1947).

[3] K.S. Novoselov, E. McCann, S.V. Morozov, V.I. Fal'ko, M.I. Katsnelson, U. Zeitler, D. Jiang, F. Schedin, A.K. Geim, Nat. Phys. 2, 177 (2006).

[4] A. Saffarzadeh, M. Ghorbani Asl, Eur. Phys. J. B 67, 239 (2009).

[5] C. Bai, J. Wang, S. Jia, Y. Yang, Physica E 43, 884 (2011).

[6] A. Yamakage, K.-I. Imura, J. Cayssol, Y. Kuramoto, Europ. Phys. Lett. 87, 47005 (2009).

[7] C.L. Kane, E.J. Mele, Phys. Rev. Lett. 95, 226801 (2005).

[8] G. Dresselhaus, M.S. Dresselhaus, Phys. Rev. 140, A401 (1965).

[9] N. Tombros, C. Jozsa, M. Popinciuc, H.T. Jonkman, B.J. van Wees, Nature (London) 448, 571 (2007).

[10] D. Huertas-Hernando, F. Guinea, A. Brataas, Phys. Rev. B 74, 155426 (2006).

[11] J.L. Cardoso, P. Pereyra, Europ. Phys. Lett. 83, 38001 (2008).

[12] M. Busl, G. Platero, Phys. Rev. B 82, 205304 (2010) J. Phys. Cond. Mat. 24, 154001 (2012).

[13] K. Vy'borny', A.A. Kovalev, J. Sinova, T. Jungwirth, Phys. Rev. B 79, 045427 (2009).

[14] J.G. Zhu, C. Park, Mater. Today 8, 35 (2006).

[15] S. Ahmadi, M. Esmaeilzadeh, E. Namvar, G. Pan, AIP Advances 2, 012130 (2012).

[16] K.S. Yi, D. Kim, K.S. Park, Phys. Rev. B 76, 115410 (2007).

[17] E.I. Rashba, Sov. Phys. Solid 2, 1109 (1960).

[18] B. Soodchomshom, Physica E 44, 579 (2011).

[19] W. Liewrian, R. Hoonsawat, I.-M. Tang, Physica E 42, 1287 (2010). 